\title{
Déficit de atención hiperactividad: un reto para el odontopediatra
}

Quijano-Villavicencio GM, Valdivieso-Vargas Machuca M. Déficit de atención e hiperactividad: un reto para el odontopediatra. Rev Estomatol Herediana. 2007;17(1):40-43.

\section{RESUMEN}

El trastorno por déficit de atención e hiperactividad (TDAH), es una patología de alta prevalencia dentro del desarrollo neurológico del niño caracterizado básicamente porque el paciente presenta una atención lábil y dispersa, impulsividad e inquietud motriz exagerada para su edad. El presente artículo describe la prevalencia, etiología, manifestaciones clínicas y tratamiento del trastorno por déficit de atención e hiperactividad (TDAH). Asimismo se presentan las consideraciones para el tratamiento odontológico incluyendo estrategias de manejo de conducta. El dentista debe considerar el comportamiento y personalidad de cada niño en particular. El objetivo principal del artículo es mostrar una guía práctica para que el odontopediatra pueda identificar a los niños con TDAH y realizar tratamiento odontológico usando técnicas de modificación de conducta, lo cual es un reto para el profesional.

Palabras clave: TRASTORNO POR DÉFICIT DE ATENCIÓN CON HIPERACTIVIDAD / ODONTOLOGÍA PEDIÁTRICA / CONTROL DE LA CONDUCTA.

Attention-deficit/hiperactivity disorder: a challenge for pediatric dentistry ABSTRACT

Attention-deficit/hiperactivity disorder (ADHD) is a high prevalence neurobehavioral disorder characterized by excessive restlessness, inattention, distraction, and impulsivity. This article describes the prevalence, aetiology, clinic characteristic and treatment of ADHD. This article presents the considerations for dental treatment including behaviour management strategies. The dentist has to considerate the child's behaviour and personality. The main objective of this article is to show a particle guide in paediatric dentistry so we can identify children with ADHD and treat them using techniques for behaviour modifications, which is a challenge for the professional.

Key words: ATTENTION DEFICIT DISORDER WITH HYPERACTIVITY / PEDIATRIC DENTISTRY / BEHAVIOR CONTROL.

\section{Introducción}

El trastorno por déficit de atención e hiperactividad (TDAH), es una patología del desarrollo neurológico del niño de alta prevalencia (1-7).

Afecta aproximadamente el 3 y $10 \%$ de los niños. Su desconocimiento hasta hace algunos años ha provocado que el TDAH este infradiagnósticado y por lo tanto mal tratado (2).

E1 TDAH es un cuadro sintomático heterogéneo desde el punto de vista clínico y pronóstico. Se caracteriza básicamente porque el paciente presenta una atención lábil y dispersa, impulsividad e inquietud motriz exagerada para su edad (1-7).

Desde el punto de vista profesional genera un elevado número de consultas médicas y es frecuente la falta de orientación del médico o psicólogo en relación a este trastorno, desde el punto de vista ambiental motiva una gran ansiedad familiar y preocupación escolar (1-7).

Esta revisión intenta recoger información de forma amplia para este tipo de trastorno conductual muy habitual en la infancia.

\section{Definición}

Este tipo de trastorno en el comportamiento ha quedado reflejado en la sociedad a través de documentos literarios y artísticos; algunos señalan a H. Hoffman como el primer autor que describe claramente a un niño con déficit de atención e hiperactividad a mediados del siglo XIX $(1,3)$.

El TDAH, término descrito desde 1970 por la Asociación Americana de Psiquiatría (APA), se define como un trastorno psicopatológico con una base biológica, que aparece a edades inferiores a los siete años de edad y que se caracteriza por tres elementos: el déficit de aten-
Gloria María Quijano Villavicencio ${ }^{1}$ Mónica Valdivieso Vargas Machuca'

'Docente del Departamento Académico de Estomatología del Niño y del Adolescente. Facultad de Estomatología. Universidad Peruana Cayetano Heredia.

\section{Correspondencia}

Gloria María Quijano Villavicencio

Pasaje Juan Parrra del Riego 254, Lima 41, Peru Teléfono: 5224213

e-mail: gloritaqv1@yahoo.com

Recibido : 13 de junio del 2007

Aceptado : 1 de diciembre del 2007

ción, la conducta hiperactiva y la impulsividad (1-6).

En la actualidad el manual de la APA clasifica el trastorno por déficit de atención con hiperactividad junto con los trastornos de comportamiento perturbador que incluyen el trastorno disocial, el trastorno negativista desafiante y el trastorno de comportamiento perturbador no especificado (7). Estos síntomas dan lugar a diferentes tipos de trastornos, tema que ahora se encuentra en plena discusión por los expertos. Se habla de dos o tres subtipos: el que se caracteriza por el predominio de la hiperactividad e impulsividad y el combinado (1-6).

El TDAH no se presenta sólo, si no que va asociado a otros trastornos que pueden ser fácilmente confundidos en las primeras etapas de la vida del niño con el propio síndrome lo que puede dificultar su diagnóstico (1-6). 


\section{Prevalencia}

Establecer con exactitud la prevalencia del TDAH se presume complejo por la diferencia de criterios empleados por los métodos y las fuentes de información, esta falta de unanimidad en la definición del problema se ha reflejado en la inclusión de pacientes dentro del TDAH, así dependiendo de las series y los métodos de diagnóstico empleado, la prevalencia de este trastorno se situaría entre el 3\% y $10 \%$ (2). Aproximadamente el 15\% de los niños con este trastorno son hijos adoptados; esta circunstancia refleja posiblemente la intensificación sintomática en pacientes predispuestos debido a una situación ambiental desfavorable (2).

La relación según el sexo, muestra mayor prevalencia en varones que en niñas. Los estudios reflejan posiciones de 1:2 a 1:6(1).

\section{Etiología}

No se conoce aún el agente o agentes etiológicos responsables de este tipo de trastorno conductual, sin embargo se han señalado diversos factores como: Influencias familiares y genéticas que involucran aspectos neuroanatómicos, neuroquímicos, neuropsicológicos; también se han considerado factores perinatales y prenatales e influencias ambientales como: el plomo y los aditivos alimenticios agentes con cierta influencia en la conducta hiperactiva (7-9).

\section{Rasgos clínicos según las dife- rentes edades $(1,2,4)$}

Nacimiento a 5 años

- Alto nivel de actividad, búsqueda de la atención, rabietas y dificultad para jugar solo.

- Desarrollo motor precoz, niños inquietos y "trepadores", con curiosidad insaciable.
- Mayor frecuencia de accidentes, heridas e intoxicaciones.

- Impacientes, insistentes y muy demandantes

- No suelen seguir las normas, molestan e interrumpen a sus compañeros.

- Poca persistencia en el juego.

6 a 12 años

- Excesiva actividad motora en clase ante la exigencia de estar sentados, pero normal en el recreo.

- Impulsividad: responden sin "escuchar", contestan por escrito sin "leer" el enunciado.

- Interrumpen la actividad y los juegos ajenos.

- Tareas escolares incompletas, no organizadas, sin hábitos de estudio y olvidos de material.

- Dificultad para el esfuerzo mental y fácil distracción por interferencias.

- Problemas de cálculo, lectura, escritura y en general del aprendizaje

Adolescencia

- Suele disminuir la hiperactividad

- Sensación interna de "inquietud" Siguen los problemas de inatención e impulsividad

- Desgaste, desmoralización y frecuentes trastornos del ánimo: hiperactivos, hipoactivos.

- Problemas de conducta

- Relación conflictiva con los adultos.

- Posibilidad de abuso de sustancias tóxicas y actividad sexual precoz y de riesgo.

- Posibilidad de abandono de los estudíos

\section{Tratamiento}

El tratamiento es multidisciplinario donde intervienen los padres de familia, el educador y el profesional de salud (10-12).

El tratamiento farmacológico es uno de los pilares de la estrategia terapéutica en estos pacientes, en nuestro medio se dispone de un medicamento estimulante metilfenidato (Ritalin), que ha sido probado a nivel mundial durante muchos años con gran eficacia (10-12).

El metilfenidato actúa elevando el nivel de alerta del sistema nervioso central, facilitando al cerebro el poder de atención y concentración; el aprendizaje y mejora en la mayoría de los casos los síntomas principales del TDAH (6,10-12).

Aunque los fármacos estimulantes son eficaces en el tratamiento de los niños con TDAH, algunos continúan teniendo dificultades emocionales, académicas o sociales a pesar del tratamiento farmacológico. En estos casos puede resultar útil combinar los fármacos con intervenciones psicosociales $(4,10)$.

Los tratamientos psicosociales de los niños con TDAH incluyen las intervenciones educativas, las escuelas de padres, los tratamientos de modificación conductual, la terapia cognitivo-conductual, el entrenamiento en habilidades sociales y la terapia familiar $(1,4,10)$.

\section{Consideraciones odontológicas}

Este tipo de alteración no viene acompañada de manifestaciones bucales particulares, sin embargo la experiencia en la práctica ha sugerido que los niños con TDAH tienden a tener un índice mayor de dientes cariados, perdidos y obturados (CPOD) que los niños sin esta condición. Esto debido a la pobre habilidad y motivación para realizar una buena higiene bucal, además los padres de estos niños gustan premiar las conductas positivas con regalos cariogénicos como las golosinas, lo que hace que esta población de niños sea un grupo de mayor riesgo a poseer caries dental (13-15).

En Otago, Nueva Zelandia, se 
realizó un estudio caso-control, determinó que el TDAH estaba asociado a una mayor probabilidad de tener altos inidices de caries dental (14). Se señala que la medicación para el tratamiento del TDAH podría quizás ser un factor de riesgo para una alta tasa de caries dental. Se requiere investigación adicional para determinar que otros factores están asociados.

Las intervenciones de promoción de salud bucal dirigidas a los niños con TDAH y a los padres; deberían promover la conciencia de que esta condición aumentará el riesgo de caries dental en estos pacientes, y por lo tanto se sugiere que medidas preventivas tales como: el cepillado dental, suplemento con flúor, y consejo dietético, pueden ser efectivas en minimizar este efecto (14).

Estrategias de manejo de conducta en el consultorio dental

Dos de los factores más importantes para el manejo del niño hiperactivo son compresión y paciencia. El manejo conductual eficaz implica, más un cambio de actitud que de técnica. 15

El odontólogo, debe lograr una armonía psicológica con las necesidades del niño en particular, si desea lograr la colaboración de este en el tratamiento dental. Hay dos razones básicas para ello: mejorar la comunicación con el paciente y brindar un modelo de actitudes y conductas para el resto del personal auxiliar (13,16-18).

El odontólogo debe tener un conocimiento activo de la psicología y mucha experiencia práctica, las cuales permitan tratar la ansiedad del paciente habitual en la consulta (1718).

Si el odontólogo no está preparado pueden aparecer sentimientos de tensión e incomodidad, pudiendo aumentar el estado de ansiedad del paciente y hasta provocar temores, lo que resulta un círculo vicioso. Para disminuir la posibilidad de este cuadro, el profesional debe estar familiarizado con las características comunes del niño de acuerdo a la edad de este, poseer información adicional como: conocer lo que mas le agrada, lo que le disgusta, que hace en sus ratos libres etc. de cada paciente en particular $(13,17,18)$.

Las metas y objetivos del examen odontológico no son distintas a las que se tienen con todos los pacientes; hay que dedicar especial atención al logro de una minuciosa historia médica y odontológica; una consulta con el médico principal del paciente puede dar importante información importante acerca del impedimento del individuo y su capacidad para desenvolverse (16).

Al igual que todos pacientes niños la primera cita suele ser la más importante, ya que preparará el campo para las citas futuras. Se debe disponer de tiempo suficiente como para hablar con los padres y el paciente antes de iniciar cualquier atención dental $(17,18)$.

Los siguientes procedimientos han sido efectivos para establecer la relación odontólogo-paciente y reducir la ansiedad en la atención odontológica $(17,18)$ :

Dar breve paseo por el consultorio antes de iniciar el tratamiento para que el paciente se familiarice con el diseño y mobiliario, así reducir el temor a lo desconocido.

Hablar lentamente y con términos sencillos. Asegurarse de que las explicaciones sean entendidas por el paciente.

Dar solo una instrucción a la vez. Felicitar al paciente luego de haber finalizado exitosamente una acción.

Mantener sesiones cortas. Progresar gradualmente a procedimientos más difíciles, luego que el pacien- te se haya familiarizado con el ambiente odontológico.

Citar a los pacientes a primeras horas del día, cuando tanto el odontólogo como el paciente se encuentran menos fatigados, tanto física como mentalmente.

En los niños con TDAH, la conducta que presentan suele verse reforzada cuanta más atención se le presta por lo tanto las conductas normales quedan desatendidas completamente; una técnica muy aceptada es cambiar estas preferencias, ignorar la mala conducta y reforzar los comportamientos adecuados. El incremento a las conductas deseables se establecen por medio de: recompensas sociales (atención, alabanzas, afecto, privilegios...) y recompensas materiales por la emisión de la conducta deseada; utilización de puntos-fichas canjeables por determinados reforzadores, si se produce la conducta-objetivo; tomar a los compañeros de clase, amigos, miembros de la familia como modelos para imitar conductas positi$\operatorname{vas}(1,2,15)$.

Además en estos niños con diagnóstico de TDAH; hay que tener en cuenta dos estrategias básicas como $(1,2,15)$ :

La relajación. Practicar períodos de relajación y autocontrol antes de empezar el tratamiento dental, a través de la respiración profunda y lenta. Se recomienda enseñar al niño a relajar sus músculos, una mayor relajación conducirá a una menor actividad muscular, ello se podrá lograr a través de masajes suaves y de técnicas como: la musicoterapia y aromaterapia.

Atención-Concentración. Se recomiendan ejercicios de razonamiento lógico, tareas de discriminación visual de estímulos gráficos. televisión, pizarra, cuentos etc. como agentes distractores; y finalmente 
juegos que capten su interés y que permitan mantener la concentración del niño mientras realizamos el tratamiento dental.

Debemos evitar en lo posible:

Concentrarnos en los aspectos negativos

Prejuzgar al niño calificándolo de desinteresado

Interpretar apresuradamente que la conducta del niño es deliberada.

Ser pesimista, podemos hacer mucho por un niño con TDAH.

\section{Conclusiones}

El TDAH es un cuadro sintomático heterogéneo desde el punto de vista clínico y pronóstico. Se caracteriza básicamente por una atención lábil y dispersa, impulsividad e inquietud motriz exagerada para la edad del niño.

E1 TDAH puede ser que influya adversamente en la salud bucal, debido a la pobre habilidad y motivación para realizarse una buena higiene bucal y a los medicamentos ingeridos.

E1 TDAH puede aumentar el riesgo de caries dental de un niño, por lo que se debería aumentar las medidas preventivas: tales como el fluoruro tópico, y monitoreo de los padres de las prácticas de higiene oral.

Una de las estrategias básicas en el manejo conductual para el tratamiento odontológico en niños con TDAH que hay que tener en cuenta es la relajación, atención y concentración.

Hay diversas técnicas de manejo de conducta que pueden permitir al odontopediatra lograr la colaboración del niño con TDAH en el tratamiento dental, lo importante es aplicar esto correctamente teniendo en cuenta el comportamiento $\mathrm{y}$ personalidad de cada niño en particular.

\section{Referencias Bibliográficas}

1. Fernandez M, Lopez M. Trastorno por déficit de atención con hiperactividad: ¡al abordaje! Rev Pediatr Aten Primaria. 2004;6(23):421-432.

2. Fernández A, Calleja B. Trastorno por déficit de atención y/o hiperactividad (TDAH). Abordaje multidisciplinar. Disponible en: http://www.med.uchile.cl/apuntes/archivos/2006/medicina/ trast_por_deficit_de_atencion_y_\% 20hiperactividad.pdf

3. Martin G. Niños con TDAH, ¿sólo traviesos?. Rev Esp Econ Salud. 2005; 4(2):59-62.

4. Higuera RF, Pella CVM. Trastorno por déficit de atención con hiperactividad: Revisión de conocimientos actuales. Act Med Gpo Ang 2004; 2(1):37-44.

5. Sell-Salazar F. Síndrome de hiperactividad y deficit de atención. Rev Neurol. 2003; 37(4):353-8.

6. Manassis K, Tannock R, Young A, Francis-John S. Cognition in anxious children with attention deficit hyperactivity disorder: a comparison with clinical and normal children. Behav Brain Funct. 2007; 3(4):1-10.

7. Cornella J, Llusent A. Trastorno por déficit de atención con o sin hiperactividad. Pediatr Integral 2003; 7(8):571-82.

8. Thapar A, O'Donovan M, Owen MJ. The genetics of attention deficit hyperactivity disorder. Hum Mol Genet. 2005;14(Spec No. 2):R275-82.

9. Matas M. Approach to attention deficit disorder in adults. Can Fam Physician. 2006; 52:961-4.

10. Rodriguez PJ. Actualización en el tratamiento del niño hiperactivo. BSCP Can Ped 2005; 29(2):39-42.

11. McLennan JD. Deciding on stimulant use for childhood ADHD: maintaining focus on the key questions. Can Fam Physician. 2006; 52:940-1, 9456.

12. Montiel-Nava C, Peña JA, Espina-Mariñes G, Ferrer-Hernández ME, López-Rubio A, PuertasSánchez S, Cardozo JJ. Estudio piloto de metilfenidato y entrenamiento a padres en el tratamiento de niños con trastorno por déficit de atenciónhiperactividad. Rev Neurol. 2002; 35(3):201-5.

13.Felicetti DM, Julliard K. Behaviors of children with and without attention deficit hyperactivity disorder during a dental recall visit. ASDC J Dent Child. 2000; 67(4):246-9, 231.

14. Broadbent JM, Ayers KM, Thomson WM. Is attentiondeficit hyperactivity disorder a risk factor for dental caries? A case-control study. Caries Res. 2004; 38(1):29-33.

15.López-Villalobos JA, RodríguezMolinero L, Sacristán-Martín AM, Garrido-Redondo M, Mártínez-Rivera T. El psicólogo clínico y el trastorno por déficit de atención con hiperactividad: vinculación con el pediatra. Rev Pediatr Aten Primaria. 2006; 8(Supl 4):S157-73.

16. Castellanos J, Gray O, Diaz L. Medicina en odontología. Manejo dental de pacientes con enfermedades sistémicas. Mexico DF: Manual Moderno; 1996.

17. Ripa L Manejo de la conducta odontológica del niño. México DF: Ediciones Mundi SAIC; 1984.

18.Pinkham JR. Behavior management of children in the dental office. Dent Clin North Am. 2000; 44(3):471-86. 